Musées, Patrimoine et Culture scientifiques et techniques

$111 \mid 2007$

mai - juin 2007

\title{
Collectivités locales : vers une différenciation aléatoire des politiques tarifaires
}

Philippe Mathieu

\section{OpenEdition \\ Journals}

Édition électronique

URL : http://journals.openedition.org/ocim/753

DOI : 10.4000/ocim.753

ISSN : 2108-646X

Éditeur

OCIM

Édition imprimée

Date de publication : 1 mai 2007

Pagination : 8-14

ISSN : 0994-1908

Référence électronique

Philippe Mathieu, «Collectivités locales : vers une différenciation aléatoire des politiques tarifaires », La Lettre de I'OCIM [En ligne], 111 | 2007, mis en ligne le 15 mars 2011, consulté le 19 avril 2019. URL: http://journals.openedition.org/ocim/753; DOI : 10.4000/ocim.753 


\section{Collectivités locales : \\ vers une différenciation aléatoire des politiques tarifaires}

Philippe Mathieu *

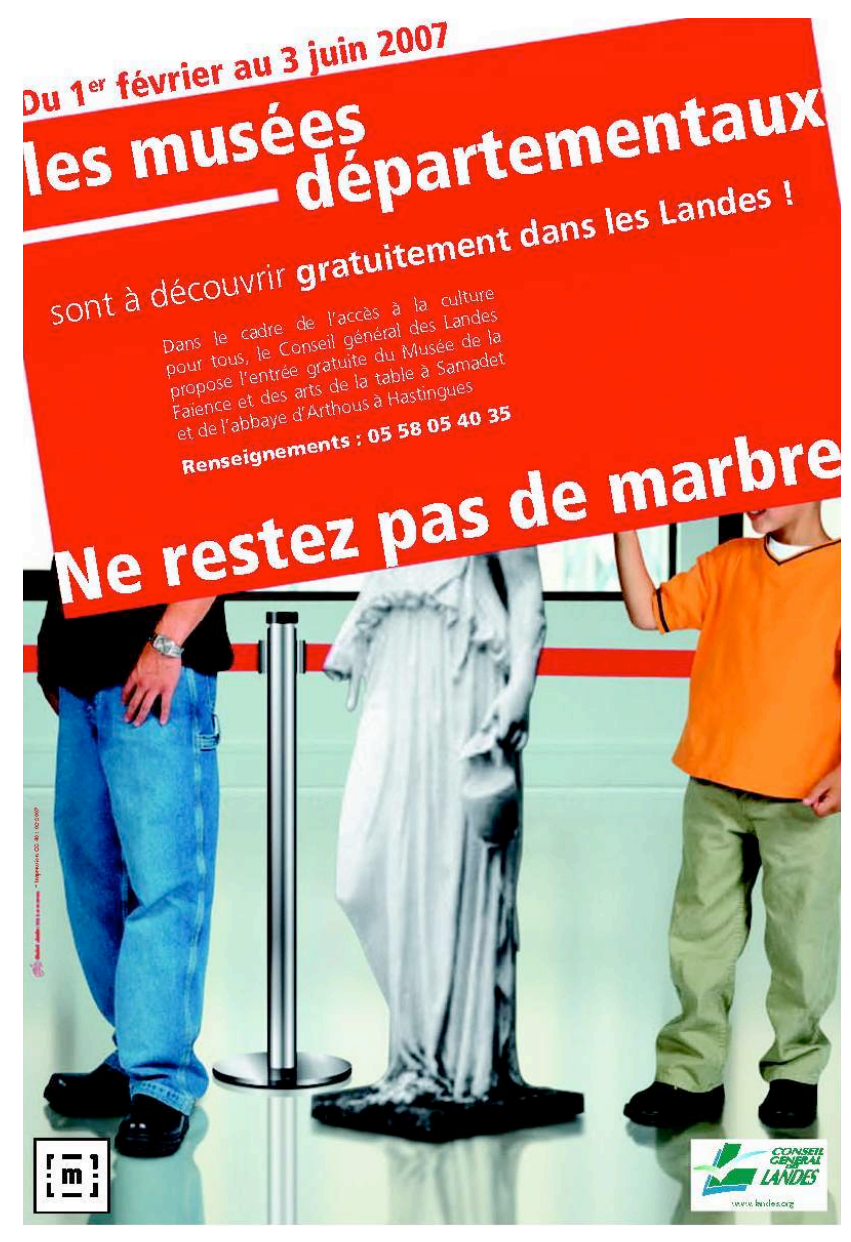

(c) Conseil général des Landes

* Philippe Mathieu est directeur de la société d'économie mixte Projet Alésia phm@alesia.com
Longtemps relativement homogènes, les politiques tarifaires des musées publics tendent désormais à se différencier. $S^{\prime}$ affranchissant des pratiques nationales autant que de leurs propres usages antérieurs, plusieurs collectivités territoriales ont en effet choisi soit de demander des droits d'entrée élevés, soit d'offrir la gratuité d'accès. Ces stratégies sont en réalité fondées sur des conceptions antithétiques $d$ 'institutions muséales pourtant toutes labellisées «Musées de France » en application de la loi du 4 janvier 2002.

« Les droits d'entrée des musées de France sont fixés de manière à favoriser leur accès au public le plus large ", indique l'article L. 442-6 du Code du Patrimoine. Mais aucun critère d'appréciation de ce principe législatif n'ayant été réglementairement défini, la grille tarifaire de tout « Musée de France » public local est en fait élaborée souverainement par la collectivité propriétaire de l'établissement en vertu du principe constitutionnel de sa libre administration. Dès lors, contrairement à l'homogénéité que l'on constate dans les musées nationaux, les droits d'entrée de ces établissements peuvent varier indépendamment de l'ampleur, de la qualité et de l'originalité des collections ou de leur présentation.

Le plus souvent, les droits d'accès aux collections permanentes sont modérés, inférieurs à ceux des grands musées nationaux. Toutefois, une évolution vers de très nettes distinctions se dessine depuis 
quelques années : tarifs en forte hausse au Mémorial de Caen (jusqu'à $18 €$ par personne) et au musée de l'Automobile de Mulhouse (10,50€); gratuité d'accès aux collections permanentes des musées des villes de Paris, Dijon et Caen (hors Mémorial), aux collections permanentes et aux expositions temporaires des musées du département de l'Isère. Quoiqu'elles ne concernent encore, à notre connaissance, qu'une trentaine des quelque 1200 «Musées de France » locaux, ces nouvelles politiques sont révélatrices des profondes mutations du rôle social, des missions et de la gestion de l'institution muséale. Aussi est-il bon d'analyser le contexte de l'émergence, puis de la mise en œuvre des nouvelles orientations avant de s'interroger sur leurs premiers effets ${ }^{(1)}$.

\section{La naissance du musée entreprise}

Créé par la ville en 1988, le Mémorial de Caen fut conçu comme le premier musée entreprise français. Positionné sur le marché du tourisme de la mémoire, géré dans le cadre d'une délégation de service public (affermage) par une société d'économie mixte (SEM), sa direction fut confiée dès l'origine non à un conservateur ou à un historien mais à un manager issu du secteur privé. Surmontant de multiples résistances, celui-ci transposa dans le champ muséal les méthodes (culture client, politique commerciale, démarche qualité, contrôle de gestion...) qu'il avait acquises au sein de grands groupes gestionnaires de restaurants et de centres de thalassothérapie. Ce choix du sénateur-maire CDS Jean-Marie Girault, président de la SEM de 1988 à 2001, fut la résultante du contexte politique de l'époque. S'étant démené des années durant pour imposer un projet à vocation civique, scientifique et touristique de 120 millions de francs considéré par beaucoup comme pharaonique et non prioritaire, cet ancien avocat témoin des bombardements de l'été 1944 opta pour un mode de gestion susceptible de limiter les coûts de fonctionnement à la charge de sa collectivité.

Avec un taux de subvention inférieur à $15 \%$, le Mémorial fait figure d'exception dans l'univers muséal français, voire européen, des années 2000. Ceci, notamment, grâce à la mise en œuvre progressive d'une politique tarifaire fondée sur une approche marketing dont le but est avant tout financier. À l'origine, les droits d'entrée se situent dans la norme : 30 F pour le plein tarif en 1988. Confronté à la nécessité de développer les ressources propres de la SEM, le directeur général, s'appuyant sur des études d'élasticité de la demande au prix, préconise des augmentations par touches successives, parallèlement à l'amélioration de l'offre culturelle et de services, de la notoriété et de l'image du lieu. Suivi par son président et son conseil d'administration, il préfère le modèle économique des parcs à thème à celui des musées, faisant fi des nombreuses critiques d'un milieu culturel dont les représentants les plus conciliants se montrent très dubitatifs quant à l'efficacité de la stratégie.

En 1996, le plein tarif est de 63 F. En 2002, il passe de $12 €$ à $16 €$ après l'ouverture de l'extension du Mémorial, puis la hausse continue. Une variation saisonnière est instaurée à partir de 2003. En 2004, pour le $60^{\mathrm{e}}$ anniversaire du Débarquement, le conseil municipal adopte un plein tarif de $18 € \mathrm{du} 1^{\text {er }}$ avril au 31 août et de $17 €$ ensuite.

Différents tarifs réduits sont appliqués : en haute saison 2004, $5 €$ pour les Caennais, entre 5 et $9 €$ pour les groupes scolaires, $14,50 €$ pour les groupes adultes, $16 €$ pour les enfants, sans compter les commissionnements de 5 à $15 \%$ accordés aux intermédiaires professionnels et les multiples opérations promotionnelles ponctuelles. De plus, face aux protestations, nombreuses, et au fait que certaines personnes font demi-tour après avoir pris connaissance du prix d'entrée, un « forfait famille » (entre 40 et 45 € selon la période) est créé début 2004 pour les vacances scolaires et les week-ends. Si bien que le plein tarif n'est payé que par 18,4\% des visiteurs durant le premier semestre 2004.

() Ville de Caen

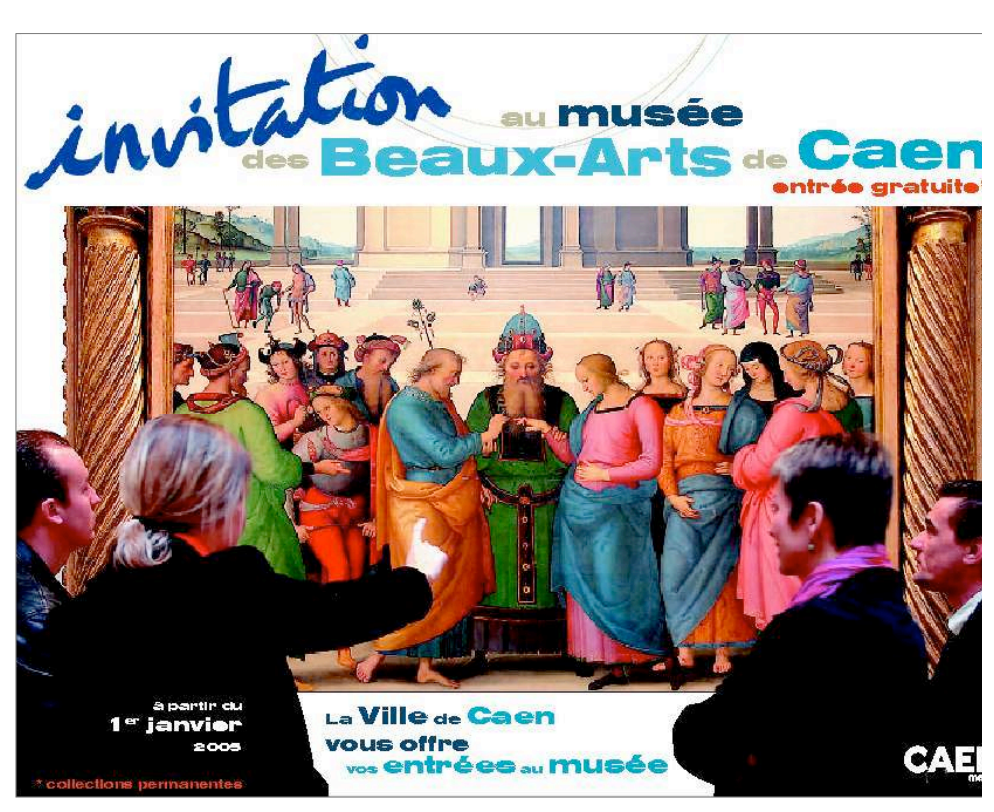


Bien qu'il soit le musée le plus cher de France avec un droit d'entrée plus de deux fois supérieur à celui des grands musées nationaux, le Mémorial de Caen est aussi le musée de province le plus visité (plus de 400000 entrées par an depuis 1999). Sa direction était néanmoins consciente, à l'époque de notre enquête, dans un contexte de difficultés budgétaires liées à une progression de la fréquentation plus faible que prévu après l'extension du musée, d'être parvenue à une limite haute sur le plan tarifaire. Le prix psychologique (dans une entreprise, prix commercialement acceptable par le plus grand nombre de clients potentiels) avait-t-il été dépassé ? Un tarif plus bas aurait-il généré une fréquentation à même de dégager une marge nette plus élevée ? La dernière étude d'élasticité remontant à 1996, il était impossible de savoir si l'optimum économique avait ou non été atteint. Toujours est-il qu'en 2005 plusieurs catégories de droits d'entrée, dont le plein tarif, ont baissé de $0,50 €$. Maintenir cette grille tarifaire, "ce qui est possible quand on a une image » et effectuer des promotions pour les familles et les groupes afin de "corriger le côté un peu élevé du prix facial » : telle était l'orientation envisagée par la direction pour les années à venir.

\section{Le virage mulhousien}

À Mulhouse, c'est en 1999 que le musée de l'Automobile est devenu un musée entreprise. Constituée dans les années 1960-1970 et ouverte au public au début des années 1980 dans des conditions rocambolesques, la prestigieuse collection Schlumpf attirait d'année en année de moins en moins de visiteurs. Parallèlement à la restauration de l'établissement, l'association gestionnaire présidée par le maire socialiste Jean-Marie Bocquel choisit alors d'en confier le management à la société privée Culture Espaces, au grand dam de la plupart des responsables culturels locaux (2). L'objectif de la ville était de faire appel à des professionnels de la gestion pour limiter le risque de devoir combler le déficit de fonctionnement d'un musée qui avait jusqu’alors vécu de ses recettes propres.

Issue de la même logique que celle du Mémorial, la politique tarifaire déterminée par Culture Espaces prend en compte trois paramètres principaux :

- l'offre de produits : " s'il y a une nouvelle attraction, un nouvel investissement, ça peut justifier une augmentation ",

- la concurrence : «on regarde ce qui se fait dans le coin »,
- l'économie générale du site : "il faut que le site retrouve l'équilibre et, à terme, dégage des bénéfices sur l'année».

Augmenté de $14 \%$ lors de la réouverture du musée après travaux en 2000 , le plein tarif est demeuré identique pendant cinq ans ( $10 €$ jusqu'en $2005 ; 10,50 €$ en 2006). En 2004, il était considéré par les responsables de l'établissement comme "assez élevé » eu égard aux commentaires des visiteurs et par rapport au prix d'une place de cinéma, sortie comparable en durée, surtout pour « la cible locale » ("les touristes passionnés d'automobile attachent moins d'importance au prix »). Prudence nourrie par une fréquentation considérablement moindre que prévu - 205000 entrées en 2004 contre 300000 projetées cinq ans plus tôt - donc des résultats financiers très en deçà des objectifs premiers. La situation s'était à ce point dégradée qu'en plus de la suppression d'un certain nombre d'emplois, la collectivité publique avait été amenée à intervenir beaucoup plus qu'elle ne l'avait envisagé au départ, entre autres en assumant annuellement, à partir de 2003, le financement de $400000 €$ de dépenses (l'assurance des collections par exemple) initialement réglées par Culture Espaces.

Au Mémorial comme au musée de l'Automobile, l'approche managériale, dont la politique tarifaire n'est que l'une des facettes, tend à assimiler la substance de l'institution à un matériau au service d'un dessein commercial. Même si le mode de gestion n'est à l'origine qu'un moyen et non une finalité, force est de constater que l'état d'esprit mercantile a sans cesse tendance à imposer sa logique propre, à transformer la structure culturelle en instrument d'une angoissante quête de clients. À Mulhouse, les mythiques témoins de l'histoire de l'automobile sont aujourd'hui utilisés comme un capital qu'il s'agit de faire fructifier au mieux en mettant en exergue son caractère exceptionnel, en racontant ses origines et en améliorant sa présentation, certes, mais aussi en lui conférant une dimension de plus en plus ludique et spectaculaire pour capter davantage de clients. À Caen, la politique éditoriale est entièrement orientée vers les ouvrages grand public. Dans les deux établissements, l'exposition temporaire n'a d'intérêt que lorsqu'elle peut être un vecteur promotionnel dont la réalisation est financièrement dénuée de risque... En d'autres termes, le défrichage des espaces culturellement épineux est aussi étranger à la nature du musée entreprise que l'état d'esprit non lucratif qui participe pourtant de la définition du «Musée de France » (3). 


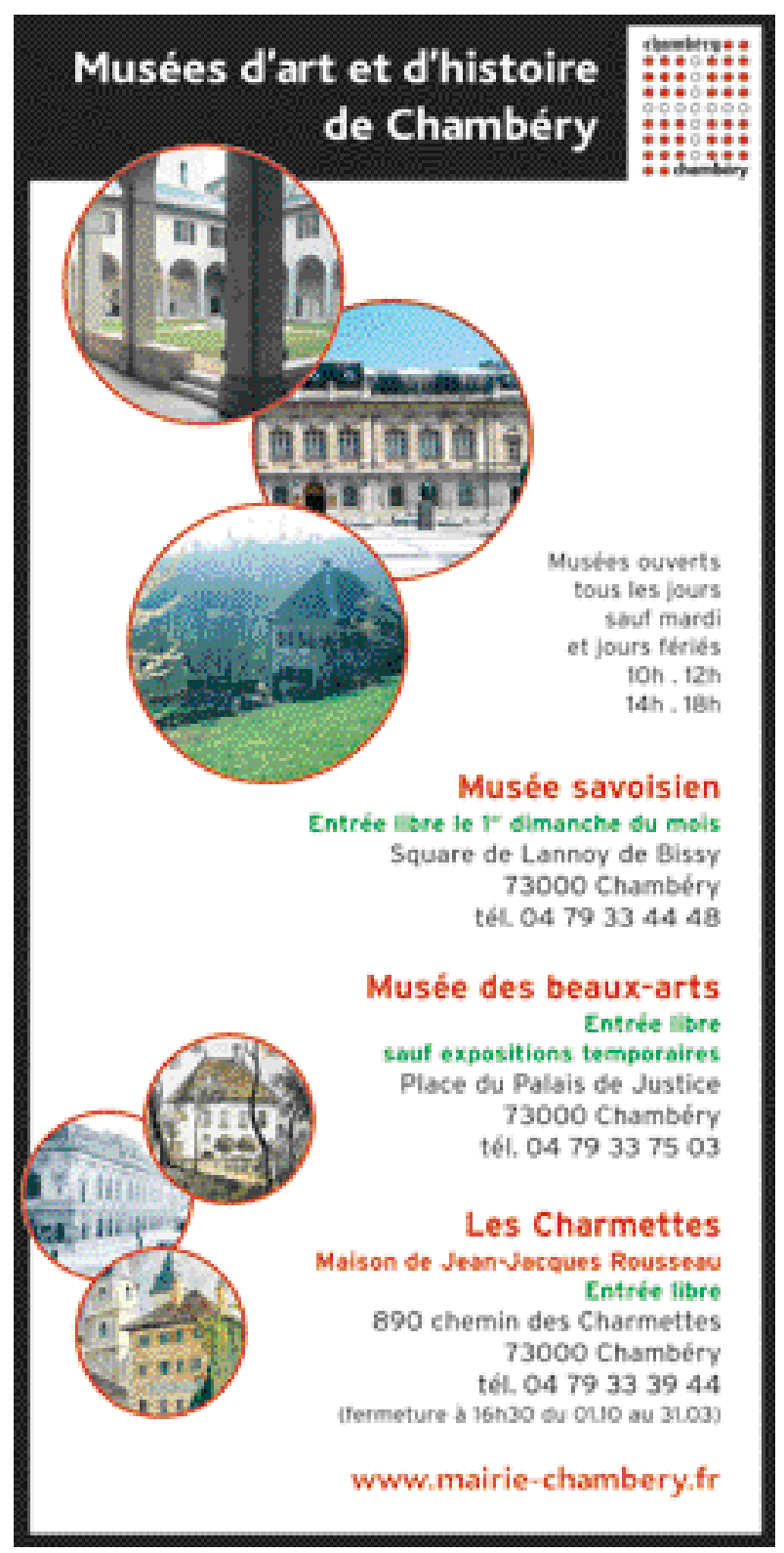

\section{La culture pour tous}

Fruit d'une réaction radicale au néo-libéralisme ambiant, la conception du bien commun et du service public qui a inspiré les promoteurs du musée gratuit est fondamentalement autre. Mis en œuvre fin 2001 dans dix musées de la ville de Paris, en 2004 dans les douze musées du département de l'Isère et les cinq musées de la ville de Dijon, en 2005 dans deux musées de la ville de Caen, la gratuité tout au long de l'année de l'accès aux collections permanentes et, en Isère seulement, aux expositions temporaires, repose sur un objectif de démocratisation culturelle.
Il s'agissait de " permettre l'accès le plus large à nos musées et en accroître la fréquentation "(Paris), " permettre l'accès à la culture de tous les Isérois partout en Isère ", "permettre l'accès à la culture au plus grand nombre »(Dijon), " mieux répondre à la mission d'éducation et de diffusion culturelle des musées et permettre l'accès à leurs collections permanentes à un plus large public »(Caen).

Malgré d'importantes plages de gratuité déjà en vigueur dans tous ces établissements, la tarification de l'accès, si modeste fût-elle, était en effet perçue par les exécutifs locaux comme un frein à la fréquentation, une barrière dressée devant les publics potentiels socialement les moins favorisés. Le fait que, depuis Bourdieu et Darbel (1966), des générations de sociologues et d'économistes avaient insisté sur l'étroite corrélation du désir de «culture cultivée » au capital culturel (Donnat, 2003), et que linfluence de la tarification s'avérait marginale sur la propension à fréquenter les musées, n'était pas perçu comme une fatalité ${ }^{(4)}$. Le volontarisme politique fondé sur une approche idéologique, jugée plus légitime que l'expertise, était censé être déterminant.

Dans la ville de droite (Caen), comme dans les trois collectivités gérées par la gauche (Paris, Dijon, Isère), la gratuité peut également être interprétée comme un symbole d'innovation. Sans doute n'estce pas un hasard si, dans les quatre cas étudiés, la décision a été prise au cours du premier mandat du leader politique local à la tête de l'exécutif. Caractéristique est à cet égard la situation de la ville de Caen où l'on trouve à la fois le musée entreprise le plus cher de France et deux musées gratuits.

L'absence de concertation entre élus et directeurs d'établissements avant le vote de la mesure - constatée à Paris, en Isère et à Caen ${ }^{(5)}$ - est révélatrice de sa motivation politique. Aussi, loin d'être tous convaincus de la pertinence de la gratuité, nombre de professionnels, mis devant le fait accompli, ont-ils manifesté peu d'engouement pour en accompagner l'application. À une exception partielle près, nous y reviendrons, aucune réflexion globale et cohérente définissant les objectifs, les moyens et les modes d'évaluation de la nouvelle politique n'avaient eu lieu au moment de notre enquête. Dans le meilleur des cas, nous avons observé l'accomplissement d'actions plutôt disparates et entendu de louables intentions. Facteur-clé de la réussite de la démocratisation, la communication fut principalement de nature politique et, dans l'ensemble, peu adaptée à la recherche de nouveaux publics. Aucun dispositif spécifique d'accueil n'avait été prévu. Quant à la conception d'actions particulières 

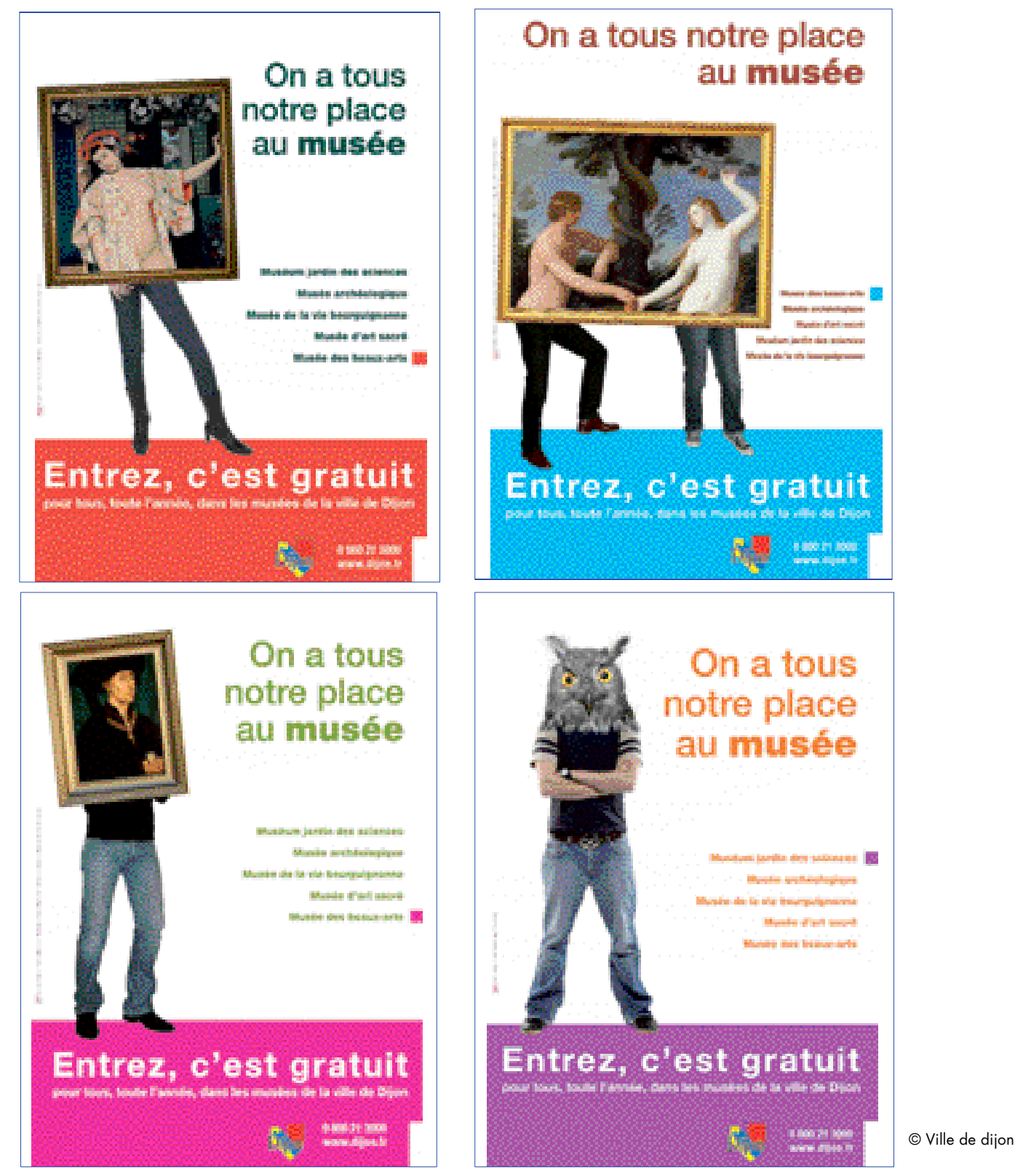

de médiation culturelle, elle ne semblait pas être au cour des préoccupations de la quasi-totalité des responsables que nous avons rencontrés.

Seule la conservation du musée des Beaux-Arts de Caen nous a paru s'investir d'emblée avec conviction dans une véritable problématique de changement. Les premières initiatives qui, au-delà de la diffusion de l'information par les vecteurs traditionnels de communication de la municipalité (relations presse, affiches, journal municipal, plaquettes), ont accompagné la mise en place de la gratuité en témoignent : distribution pour la première fois de la brochureprogramme quadrimestrielle du musée dans 40000 boîtes aux lettres de l'agglomération, mise en place de visites guidées et d'ateliers dont les thèmes et les horaires étaient susceptibles d'intéresser un public plus large que celui des habitués. Utilisée comme levier d'une revitalisation de la politique muséale, l'entrée en vigueur de la gratuité devait d'ailleurs être prolongée par le recrutement d'un responsable des publics et de nouveaux guides conférenciers.

Cela étant, nulle part la gratuité d'accès n'est aujourd'hui totale. À des degrés divers, chacune des collectivités concernées a adopté un compromis entre volonté de démocratisation par la suppression de la barrière tarifaire et impératif de rigueur budgétaire. Partout, sauf en Isère, l'entrée des expositions temporaires est payante et souvent le tarif est relativement élevé, jusqu'à $9 €$ à Paris en 2006. De plus, dans tous les musées certaines animations sont tarifées, y compris en Isère pour les visites guidées. 


\section{Des effets ambivalents}

Ces deux conceptions antithétiques de la politique tarifaire et, plus globalement, de l'institution muséale produisent-elles les effets attendus par leurs initiateurs ? Si le caractère récent de leur mise en œuvre et l'inexistence d'études de publics adéquates invitent à une certaine circonspection, les conséquences observables se révèlent ambivalentes.

Une analyse détaillée des statistiques parisiennes et iséroises associée à des entretiens avec les personnels d'accueil montre que la gratuité, tout en ayant un impact sur la fréquentation, n'est pas systématiquement et durablement à l'origine d'une augmentation présentée comme spectaculaire par les porteparole des collectivités. D'une part tous les musées ne sont pas concernés par la hausse, d'autre part d'autres facteurs peuvent expliquer les croissances les plus fortes.

Ainsi, la modification de l'entrée du musée Carnavalet, à Paris, est-elle probablement à l'origine d'une partie au moins du quasi triplement des visites en 2002, augmentation qui représentait $85 \%$ de la hausse de $61 \%$ enregistrée pour l'ensemble des musées parisiens. De même, l'exposition temporaire Trésors d'Egypte au musée Dauphinois explique-telle une part substantielle de la progression de $69 \%$ de la fréquentation globale des musées du département de l'Isère en 2004.

En outre, l'évolution sur une plus longue période forte hausse les premières années, réduction ensuite à Paris $(-35 \%$ en 2004) comme en Isère (- $21 \%$ en 2005) - incline à prendre en considération l'effet lune de miel identifié par Anne Gombault : « Le passage à la gratuité crée un événement, suscite de linntérêt. C'est cet événement, et non pas la gratuité en tant que telle, qui provoque une augmentation de la fréquentation. On sait aussi - toutes les études faites par des économistes au Canada, aux États-Unis, en Italie, en Angleterre... le démontrent - qu'à terme, la fréquentation redevient identique à ce qu'elle était avant si on ne fait rien de plus et que la structure de la fréquentation est la même. La gratuité ne fidélise pas les publics si on ne propose rien d'autre » (6).

Quant au financement du musée par ses seuls visiteurs, les expériences conduites à Caen et à Mulhouse montrent ses limites, aucun des deux musées n'étant indépendant de la manne publique, et prouvent qu'elle inscrit l'institution dans une logique de marché gauchissant ses missions culturelles et pédagogiques au bénéfice d'une course à la

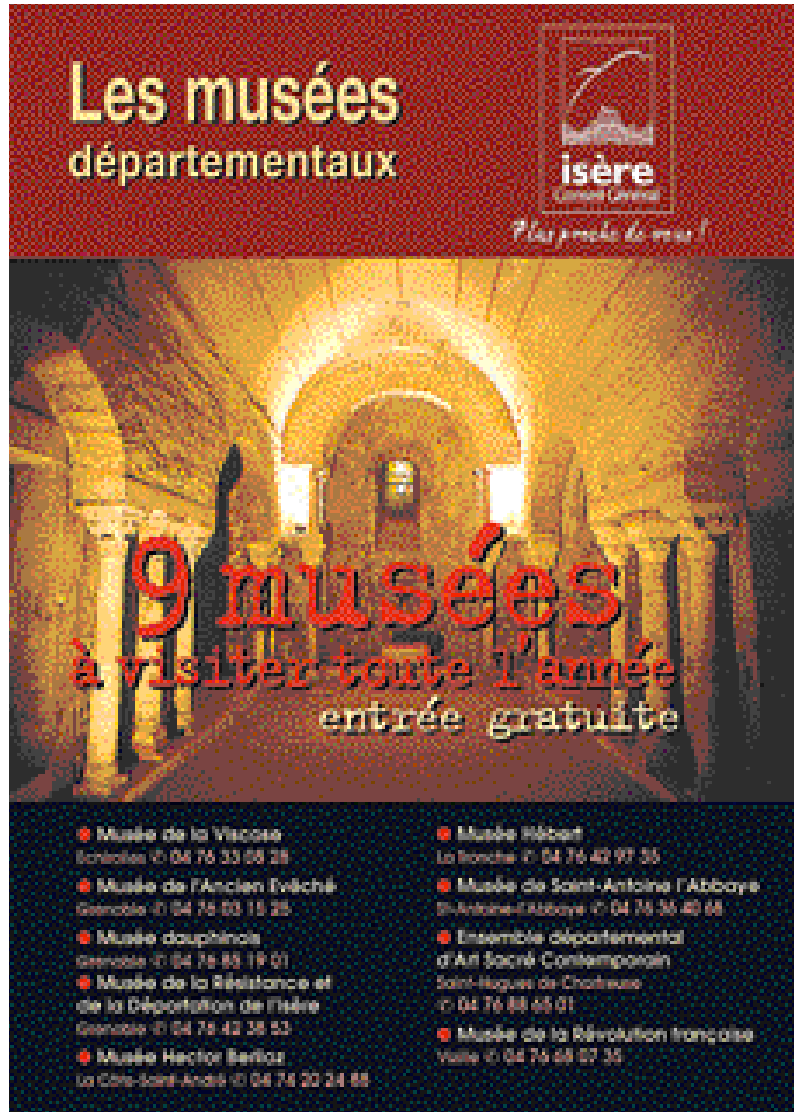

() Conseil général de l'lsère

rentabilité à court terme. L'attention de ses dirigeants n'est plus tournée vers l'enrichissement, la restauration, l'étude et la présentation au public des collections dans une perspective de démocratisation culturelle ; elle est orientée vers le développement d'activités devant a priori plaire au plus grand nombre de leurs cibles, l'optimisation des techniques de vente et l'amélioration de la marge nette. Ceci étant, la tarification élevée ne se traduit pas par une chute très sensible de fréquentation, ce qui accrédite la théorie de la faible élasticité de la demande par rapport au prix généralement défendue par les économistes de la culture.

Cette constatation conforte également l'hypothèse selon laquelle les promoteurs de la gratuité, en imaginant qu'elle serait un sésame, ont assurément minimisé la force d'inertie des habitudes grandement influencées par les origines sociales et le parcours éducatif de chacun. La figure du musée gratuit n'est pas pour autant à classer purement et simplement parmi les tentatives illusoires d'élargissement des publics d'une institution qui n'attire au moins une fois l'an que le tiers des Français. À condition qu'elle s'accompagne d'un véritable questionnement sur le positionnement et les actions du 
musée en direction de ses « non-publics » et de ses publics occasionnels, elle peut être le catalyseur d'une (r)évolution dont les fruits mûriront peu à peu. Cela suppose des moyens supplémentaires ou, à tout le moins dans les établissements les mieux dotés, une réaffectation à cet effet d'une partie des moyens existants. Cela présuppose une conviction et une volonté d'action convergente et durable de la part des décideurs politiques et professionnels.

De son côté, le musée entreprise peut être appréhendé en tant qu'établissement sui generis tenant à la fois du parc de loisirs et du musée. Une figure hybride particulièrement tonique dont les responsables s'activent à la mesure du défi auquel il sont confrontés : développer son attractivité pour assurer sa pérennisation. Son autofinancement s'avérant malgré tout inatteignable, sa survie dépend aussi du subventionnement de la collectivité propriétaire. La tension permanente inhérente à cette configuration, si elle ne saurait à notre sens constituer la base solide d'un nouveau modèle de "Musée de France ", permet de mesurer l'intérêt d'une transposition dans le champ muséal de méthodes qui conditionnent la performance des entreprises du secteur privé. Du marketing au management par objectifs en passant par le contrôle de gestion, ces outils ne sont certainement pas à rejeter en bloc en estimant qu'ils conduisent inéluctablement le musée à sa perte. Dans une période de raréfaction des fonds publics, utilisés à bon escient, ils peuvent au contraire conforter le projet d'un établissement fermement ancré dans une ligne scientifique et culturelle en phase avec une société en profonde mutation, initié ou validé par le politique, porté par des professionnels qui sont les garants de son respect.

Partis du constat de la diversification récente des politiques tarifaires, nous sommes arrivés à celui d'un relativisme du concept de musée public qui rejoint d'autres signes avant-coureurs d'un diagnostic plus global de la formation, avec la décentralisation, d'une «démocratie différentielle ${ }^{(7)}$, autrement dit d'une territorialisation de l'action publique. Relativisme des effets produits par la relation triangulaire qui s'instaure entre les élus, les professionnels et les publics (usagers). Relativisme des dogmes de service public portés par les responsables politiques, qu'il s'agisse de la commercialisation salvatrice ou de la gratuité libératrice. Relativisme de l'isomorphisme des milieux professionnels pour encadrer la montée du client-centrisme, la standardisation des pratiques étant moins souvent à l'ordre du jour. Relativisme des enjeux de citoyenneté différemment appréciés d'une configuration territoriale à une autre. Au pays de la "démocratie différentielle», l'harmonisation du statut des "Musées de France » n'est sans doute qu'utopie.

\section{Notes}

(1) L'étude de terrain qui a servi de base à la rédaction de cet article a été réalisée entre mars 2004 et avril 2005.

(2) Les collections ont toutefois été placées sous la responsabilité d'un conservateur non rattaché à la société privée.

(3) Le Mémorial et, dans une bien moindre mesure, le musée de l'Automobile ne sauraient cependant être réduits à cette pure nature. Une analyse plus complète de leurs missions, de leurs activités et de leur fonctionnement mettrait en lumière leur action en matière scientifique, culturelle et pédagogique.

(4) L'expérience de la gratuité du premier dimanche du mois au musée du Louvre (lire par ailleurs) peut inciter à nuancer cette affirmation. Mais, outre qu'il ne s'agit pas d'une gratuité permanente, le musée du Louvre, du fait de sa spécificité, n'est-il pas l'exception qui confirme la règle ?

(5) Nous n'avons pas étudié cette question à Dijon.

(6) Gombault, A. L'émergence du prix comme variable stratégique des musées, in Rouet (2002), pp. 165-221. La citation est extraite de l'intervention de l'auteur lors du Forum Ptolémée le 19 octobre 2004 à la Cité des Sciences et de l'Industrie.

(7) Faure, A. et Mathieu, P. Le service public, les leaders politiques locaux et les clients-citoyens, le cas d'école des musées gratuits et des musées entreprises, communication (non encore publiée) dans le cadre du quinzième colloque de la revue Politiques et Management Public, «L'action publique au risque du client ? Client-centrisme et citoyenneté », Science-Po Lille, 16-17 mars 2006.

\section{Bibliographie}

Donnat, O. (dir.) Regards croisés sur les pratiques culturelles. Paris : La Documentation française, 2003, 348 p.

Mathieu, P. Émergence de nouvelles figures muséales. «Musée gratuit » versus «Musée entreprise ", Mémoire, Master «Direction de projets culturels », université Pierre Mendès France, institut d'Études politiques de Grenoble, Observatoire national des Politiques culturelles, ministère de la Culture et de la Communication, 2005, 169 p.

Rouet, F. (dir.) Les tarifs de la culture. Paris : La Documentation Française, 2002, 383 p.

Tobelem, J.-T. Le nouvel âge des musées. Les institutions culturelles au défi de la gestion. Paris : Armand Colin, 2005, 318 p. 